\title{
Datenerhebung: Histrelin-Therapie unter Praxisbedingungen
}

— Seit April 2012 können Mitglieder des Interessenverbandes zur Qualitätssicherung der Arbeit niedergelassener Uro-Onkologen in Deutschland e. V. (IQUO) an einer neuen, nicht interventionellen Studie zur Therapie des fortgeschrittenen und metastasierten Prostatakarzinoms mit dem Histrelin-Jahresimplantat $\left(\right.$ Vantas $\left.^{\oplus}\right)$ teilnehmen. Ziel der VIEW (Vantas ${ }^{\oplus}$ - Insights and Experiences in an Alternative Way of Treatment)-Studie ist es, Behandlungsdaten in dieser Indikation unter den realen Bedingungen der uro-onkologischen Versorgung zu erheben. In Kooperation mit Orion Pharma können alle IQUOMitglieder noch bis Ende März 2013 Pati- enten, die für eine LHRH-Therapie infrage kommen, in diese Studie einschließen.

Die prospektive Registerstudie im IQUO e. V. protokolliert den therapeutischen Effekt der Hormon-Suppressionstherapie mit dem Histrelin-Jahresimplantat über einen Zeitraum von 15 Monaten. Bei allen Teilnehmern wird zu Beginn auf der Innenseite des Oberarms das Vantas ${ }^{\circledR}$-Jahresimplantat eingesetzt. Nach zwölf Monaten entscheidet der Arzt entsprechend des klinischen Befundes, ob die Therapie mit einem neuen Implantat fortgesetzt wird oder sogar eine Behandlungspause eingelegt werden kann. Insgesamt sind acht Untersuchungstermine vorgesehen. Unter anderem wird dabei die Lebensqualität mithilfe des PORPUS (Patient Oriented Prostate Cancer Utility Scale)-Fragebogens zu Beginn, nach zwölf und nach 15 Monaten ermittelt. Ferner werden mithilfe des IQUO-verbandseigenen Dokumentationssystem QuaSi ${ }^{\circledast}$ Uro klinische Parameter dokumentiert (z.B. Beeinflussung des Testosteronspiegels unter kontinuierlicher Therapie, PSA-Wert-Verlauf). Weitere Informationen erhalten interessierte IQUO-Mitglieder über die IQUO-Geschäftsstelle und den Außendienst von Orion Pharma.

Nach Informationen von

Orion Pharma, Hamburg

\section{Skelettale Komplikationen bei Knochenmetastasen verzögern}

— Dem therapeutischen Fortschritt verdanken Tumorpatienten ein erheblich längeres Überleben. Die Patienten sollten die gewonnene Lebenszeit mit einer guten Lebensqualität verbinden können, betonte Prof. Petra Feyer, Berlin. In der palliativen Situation sind die Erhaltung der Lebensqualität, die Linderung tumorbedingter Symptome und die Verzögerung vital bedrohlicher Komplikationen die wichtigsten Therapieziele. So sollten die Patienten vor den Folgen von Knochenmetastasen geschützt werden, die im Rahmen zahlreicher solider Tumorerkrankungen auftreten (z.B. bei 65-70\% der Patienten mit Mamma- und Prostatakarzinom) und mit hohem Risiko skelettaler Komplikationen wie Knochenschmerzen, pathologische Frakturen oder Rückenmarkskompression assoziiert sind.

Patienten mit Knochenmetastasen profitieren von einer osteoprotektiven Therapie, wie mit dem RANKL (Receptor Activator of NF-KB-Ligand) Denosumab, XGEVA ${ }^{\oplus}$ ), sagte Prof. Ingo Diehl, Mannheim. Die Zulassung von Denosumab basiert auf drei randomisierten Studien, in denen insgesamt 5.723 Patienten mit Knochenmetastasen entweder mit s.c. Denosumab/ i.v. Placebo oder i.v. Zoledronsäure/s.c. Placebo behandelt wurden [Lipton A et al. ESMO, 2010 Abstract 1249P]. Denosumab verlängerte die Zeit bis zur ersten Skelett-bezogenen Komplikation signifikant um zusätzliche $8,2$ Monate ( 27,6 vs. 19,4 Monate, $\mathrm{p}<0,0001)$ und reduzierte das Risiko skelettaler Komplikationen gegenüber Zoledronsäure um weitere $17 \%$, berichtete Diehl. Die Zeit bis zur Schmerzzunahme oder zum Auftreten moderater/starker Schmerzen wurde um 55 Tage verlängert (143 vs. 198 Tage). In den aktuellen Leitlinien der Arbeitsgemeinschaft Gynäkologische Onkologie (AGO) wird Denosumab aufgrund dieser Ergebnisse mit dem höchsten Empfehlungsgrad (++) bewertet.

Dr. Günter Springer

Medien-Forum „Knochen \& Krebs. Je früher desto besser: Effektive Prävention von skelettalen Komplikationen von Anfang an",

4. Mai 2012, München

Veranstalter: Amgen, München
Intravesikale Therapieoption bei chronischen Zystitiden

- Eine kausalnahe Behandlung chronischer Zystitiden verspricht die GAG-Ersatztherapie mit Gepan ${ }^{\circledast}$ instill, so das Expertenfazit auf dem Satelliten-Symposium „Chronische Zystitiden: Ursachen - Diagnose - innovative Therapieoptionen“ in Hamburg. Die intravesikale Instillation mit Gepan ${ }^{\circledast}$ instill versorgt die Glycosaminoglycan-(GAG)-Schicht mit ihrem Baustein Chondroitinsulfat und behebt den Defekt im Urothel. Davon können Patienten aller Formen der chronischen Zystitis (interstitielle Zystitis = IC, überaktive Blase, Radiogene Zystitis, chronisch-rezidivierende Harnwegsinfekte) profitieren. Wenn medikamentöse Therapien mit Pentosanpolysulfat, Antihistaminika oder Antidepressiva ausgeschöpft sind, sei die intravesikale Instillationstherapie laut Prof. Daniela SchultzLampel, Villingen-Schwenningen, eine wichtige Option: „Hier können Erfolgsraten bis zu $80 \%$ erwartet werden“. Dies zeigte auch eine multizentrische prospektive Beobachtungsstudie an 286 Patienten mit chronischen Zystitiden [Nordling J, van Ophoven A. Drug Research 2008]. 77\% der 165 IC-Patienten bewerteten die Behandlung positiv: Harndrang, Miktionsfrequenz am Tag, Nykturie sowie Blasenschmerzen wurden signifikant gebessert.

Bei der intravesikalen Applikation sollte das Volumen ausreichend groß sein, um eine gute Benetzung des Urothels zu gewährleisten. Prof. Helmut Madersbacher, Innsbruck, sagte: "Sehr gute Therapieerfahrungen gibt es hier zur Instillation einer 0,2\%igen Chondroitinsulfatlösung mit einem Volumen von $40 \mathrm{ml}$.

Nach Informationen von

Pohl-Boskamp, Hohenlockstedt 